\title{
Bhageerath-Targeting the near impossible: Pushing the frontiers of atomic models for protein tertiary structure prediction ${ }^{\#}$
}

\author{
B JAYARAM ${ }^{\mathrm{a}, \mathrm{b}, \mathrm{c}, *}$, PRIYANKA DHINGRA ${ }^{\mathrm{a}, \mathrm{b}}$, BHARAT LAKHANI $^{\mathrm{b}}$ \\ and SHASHANK SHEKHAR ${ }^{b}$ \\ a Department of Chemistry, ${ }^{\mathrm{b}}$ Supercomputing Facility for Bioinformatics and Computational Biology, \\ ${ }^{\mathrm{c}}$ School of Biological Sciences, Indian Institute of Technology Delhi, Hauz Khas, New Delhi 110016, India \\ e-mail: bjayaram@chemistry.iitd.ac.in
}

\begin{abstract}
Protein folding, considered to be the holy grail of molecular biology, remains intractable even after six decades since the report of the first crystal structure. Over 70,000 X-ray and NMR structures are now available in protein structural repositories and no physico-chemical solution is in sight. Molecular simulation methodologies have evolved to a stage to provide a computational solution to the tertiary structures of small proteins. Knowledge base driven methodologies are maturing in predicting the tertiary structures of query sequences which share high similarities with sequences of known structures in the databases. The void region thus seems to be medium (>100 amino acid residues) to large proteins with no sequence homologs in the databases and hence which has become a fertile ground for the genesis of hybrid models which exploit local similarities together with ab initio models to arrive at reasonable predictions. We describe here the development of Bhageerath an ab initio model and Bhageerath-H a hybrid model and present a critique on the current status of prediction of protein tertiary structures.
\end{abstract}

Keywords. Ab initio; protein folding; molecular dynamics simulation; protein structure prediction; Bhageerath; critical assessment of protein structure prediction (CASP).

\section{Introduction}

Protein folding, considered to be a challenging task ${ }^{1-3}$ remains unsolved for the last six decades. It is classified as an NP complete or NP hard problem. ${ }^{4,5}$ This notwithstanding, the dire need for tertiary structures of proteins in drug discovery and other areas ${ }^{6-8}$ has propelled the development of a multitude of computational recipes. In this article, we focus on ab initio/de novo strategies, Bhageerath in particular, for protein tertiary structure prediction.

The $a b$ initio term in the context of protein structure prediction is used to signify the usage of physics based all atom molecular mechanics potentials for predicting the three-dimensional structure of a protein. Molecular dynamics, Monte Carlo simulations and their variants are pooled under this category. Molecular dynamics simulations, in particular, have provided extremely high resolution spatial and temporal data, enhancing our knowledge and understanding of the protein folding mechanism. Simulations of biologically relevant processes, with atomistic accuracy on timescales beyond

\footnotetext{
\#Dedicated to Prof. N Sathyamurthy on his 60th birthday

*For correspondence
}

microsecond are now possible due to advances in software and hardware. ${ }^{9}$

In the year 1975, Levitt and Warshel simulated the folding of bovine pancreatic trypsin inhibitor (BPTI) using a simple representation of protein conformation, energy minimization and thermalization. They succeeded in 'renaturing' the protein from an open fully extended conformation to a folded native like conformation. ${ }^{10}$ Later in 1977, McCammon, Gelin and Karplus studied for the first time the dynamics of folded BPTI in vacuum at a molecular level over a period of 9.2 picoseconds. ${ }^{11}$ Since then extensive research has been carried out in the area of protein folding, unfolding, dynamics and structure. Table 1, summarizes some major milestones capturing the advancements. In the year 1995, Li and Daggett studied the structure and dynamics of native chymotrypsin inhibitior 2 with explicit water from a $5.3 \mathrm{~ns}$ simulation. ${ }^{12}$ Insights were gained from $550 \mathrm{ps}$ unfolding simulations of reduced BPTI at high temperature. ${ }^{13}$ Folding simulation of small peptide fragments such as beta-turn in a short linear peptide and $\beta$-heptapeptides in aqueous solution were performed for $20 \mathrm{~ns}$ and $50 \mathrm{~ns} .{ }^{18,19}$ Using a Cray T3E, a massively parallel supercomputer consisting hundreds of CPUs, Duan and Kollman 
Table 1. Increasing length of simulations with advances in computing resources.

\begin{tabular}{|c|c|c|c|}
\hline Sl. No. & System & Length of the simulation & Year \\
\hline 1 & Bovine Pancreatic Trypsin Inhibitor (BPTI) ${ }^{11}$ & $9.2 \mathrm{ps}$ & 1977 \\
\hline 2 & Bovine Pancreatic Trypsin Inhibitor (BPTI) ${ }^{14,15}$ & $60 \mathrm{ps}, 132 \mathrm{ps}$ & 1983 \\
\hline 3 & Bovine Pancreatic Trypsin Inhibitor (BPTI) ${ }^{13}$ & $550 \mathrm{ps}$ & 1992 \\
\hline 4 & Apomyoglobin ${ }^{16}$ & $350 \mathrm{ps}, 500 \mathrm{ps}$ & 1993 \\
\hline 5 & Chymotrypsin inhinitor $2(\mathrm{CI} 2)^{12}$ & $5.3 \mathrm{~ns}$ & 1995 \\
\hline 6 & Staphylococcal protein ${ }^{17}$ & $>9 \mathrm{~ns}$ & 1995 \\
\hline 7 & Pentapeptide cis-AYPYD $^{18}$ & $20 \mathrm{~ns}$ & 1997 \\
\hline 8 & $\beta$-Heptapeptide ${ }^{19}$ & $50 \mathrm{~ns}$ & 1998 \\
\hline 9 & Villin headpiece ${ }^{20}$ & $1 \mu \mathrm{s}$ & 1998 \\
\hline 10 & Engrailed Homeodomain $^{21}$ & $40 \mathrm{~ns}, 70 \mathrm{~ns}$ & 2000 \\
\hline 11 & Protein $G^{22}$ & $38 \mu \mathrm{s}$ & 2001 \\
\hline 12 & Trp cage ${ }^{23}$ & $50 \mathrm{~ns}$ & 2002 \\
\hline 13 & Trp cage ${ }^{24}$ & $\sim 100 \mu \mathrm{s}$ & 2002 \\
\hline 14 & Human Pin1 WW domain mutant, FiP35 25,26 & $10 \mu \mathrm{s}$ & 2009 \\
\hline 15 & NTL9 $^{27}$ & $1.52 \mathrm{~ms}$ & 2010 \\
\hline \multirow[t]{2}{*}{16} & FiP35, Villin headpiece ${ }^{28}$ & $100 \mu \mathrm{s}$ & 2010 \\
\hline & Bovine Pancreatic Trypsin Inhibitor (BPTI) & $1 \mathrm{~ms}$ & \\
\hline
\end{tabular}

in 1998 reported one of the longest simulations of that time for a protein in water. They simulated villin headpiece subdomain (HP-36) for $\sim 1 \mu$ s with $\sim 3000$ water molecules. ${ }^{20}$ The growing track record of protein folding simulations in a high performance computing environment stimulated IBM to announce in December 1999, a five year effort to build a massively parallel computer 'Blue Gene' to study biomolecular phenomena. ${ }^{29}$ In 2000, protein unfolding simulations of Engrailed Homeodomain (En-HD) from Drosophila melanogaster a 61 residue, mainly $\alpha$-helical protein was carried out for a maximum time scale of $70 \mathrm{~ns} .^{21}$ The introduction of distributed computing paved the way towards achieving longer time scales in the simulations of the dynamics of biomolecules at atomic level. ${ }^{30}$ The Folding@ home project used distributed computing techniques and a super cluster of thousands of computer processors to simulate $38 \mu \mathrm{s}$ of folding time. Folding of the $\mathrm{C}$-terminal $\beta$-hairpin from protein $\mathrm{G}$ in atomistic detail using the GB/SA implicit solvent model at $300 \mathrm{~K}$ was reported. ${ }^{22}$ Later in 2001 all atom protein structure prediction using $a b$ initio protein folding simulation was carried out for trpcage TC5b with extended initial conformation for a time period of $50 \mathrm{~ns}$ at $300 \mathrm{~K} .{ }^{23} \mathrm{Simu}-$ lations for Trp-cage for an aggregate time of $\sim 100 \mu \mathrm{s}$ were performed by the Folding@home project to capture the rapid relaxation from an extended starting state to a relaxed unfolded state. ${ }^{24}$ In 2003 , Langevin dynamics was applied to the physics based united residue (UNRES) force field to generate trajectories for seven proteins with an average folding time of the order of nanoseconds. Folding with Langevin dynamics helped in exploring thousands of folding pathways and also enabled predicting not only the native structure but also the folding scenario of the protein. ${ }^{31}$ The improved performance of molecular dynamics softwares and computing resources made it possible to perform multiple microsecond simulations in explicit solvent environment. Using the high performance computing machines Ensign et al. in 2007 presented large folding trajectories for villin mutant. ${ }^{32}$ Freddolino et al . reported in 2008, a $10 \mu$ s trajectory of the fast folding human Pin1 WW domain mutant Fip35. ${ }^{25,26}$ A recent initiative by Folding@ home distributed computing platform was successful in performing a large array of distributed implicit solvent folding simulations of a 39 residue protein NTL9(1-39) using Amber ff96 force field and accelerated version of GROMACS for GPU processors for an aggregate time scale of $1.52 \mathrm{~ms} .^{27}$ Crossing the barriers of computational resources, Shaw et al. developed a special purpose machine christened 'Anton', which has greatly accelerated the execution of simulations and generation of trajectories of $1 \mathrm{~ms}$ length. Such massively parallel specialized machines have allowed all-atom molecular dynamics simulations of proteins in an explicit solvent environment at a much faster rate and 100 times longer time scales. ${ }^{28}$ Taking the protein folding in a new direction, researchers at the University of Washington developed a protein folding video game Foldit that uses human visual problem solving and strategy development capabilities with traditional computing algorithms. ${ }^{33}$ 
Despite the significant advantages and power of molecular simulations, the field of $a b$ initio protein folding still faces serious challenges. The huge amount of sampling space and the deficiencies in potential functions restrict the use of simulations to smaller proteins and refinement of models produced by low-resolution methods. ${ }^{34}$ Increasing availability of experimentally determined protein structures has inspired the development of knowledge based methods for structure prediction. With the exception of 'pure' physico-chemical approaches, ${ }^{35}$ these methods rely on searching the protein structure databases and using the available structural information to predict the tertiary structure of new sequences. The bi-annual community wide Critical Assessment of Protein Structure Prediction (CASP) experiments ${ }^{36,37}$ classify such methods under the category of Template based modelling. The term $a b$ initio is used in much broader sense in CASP and includes methods that compare fragments (short stretches) of query sequence of unknown structure with sequences in protein structure database (RCSB) ${ }^{38}$ and assemble atomic models for the whole protein with varying strategies for dealing with regions with missing matches. The primary obstacle to these template based methods is database dependency especially where a related structural homolog is not available or where the query sequence presents a totally new fold. Providing an understanding of the forces driving the protein structure formation is obviously beyond the purview of these methods. ${ }^{39}$

\section{Methodology}

In a modification of the $a b$ intio procedures delineated above (called de novo methods which use partial information from structural databases) as for instance using database searches for secondary structures only and no use of database information for the tertiary structure prediction, we describe here an improved and computationally robust version of Bhageerath ${ }^{40}$ an energy based software suite for narrowing down the search space of tertiary structures of small globular proteins. The protocol comprises eight different computational modules that form an automated pipeline. Proceeding from the input amino acid sequence, the software first predicts the secondary structure information (helix/strand/loop) along the entire length of the protein. The second module creates an atomic-level extended structure using the secondary structure information. The third module generates a large number of trial structures with a systematic sampling of the conformational space of loop dihedrals. The number of trial structures generated is $128^{(n-1)}$ where ' $n$ ' is the number of secondary structural elements and ' $n-1$ ' is the number of loops/junctions between the secondary structural units. These structures are generated by choosing seven dihedrals from each of the loops (three at both ends and one dihedral from the middle of the loop) and sampling two conformational states for each dihedral. The generated trial structures are screened in the fourth module through persistence length, radius of

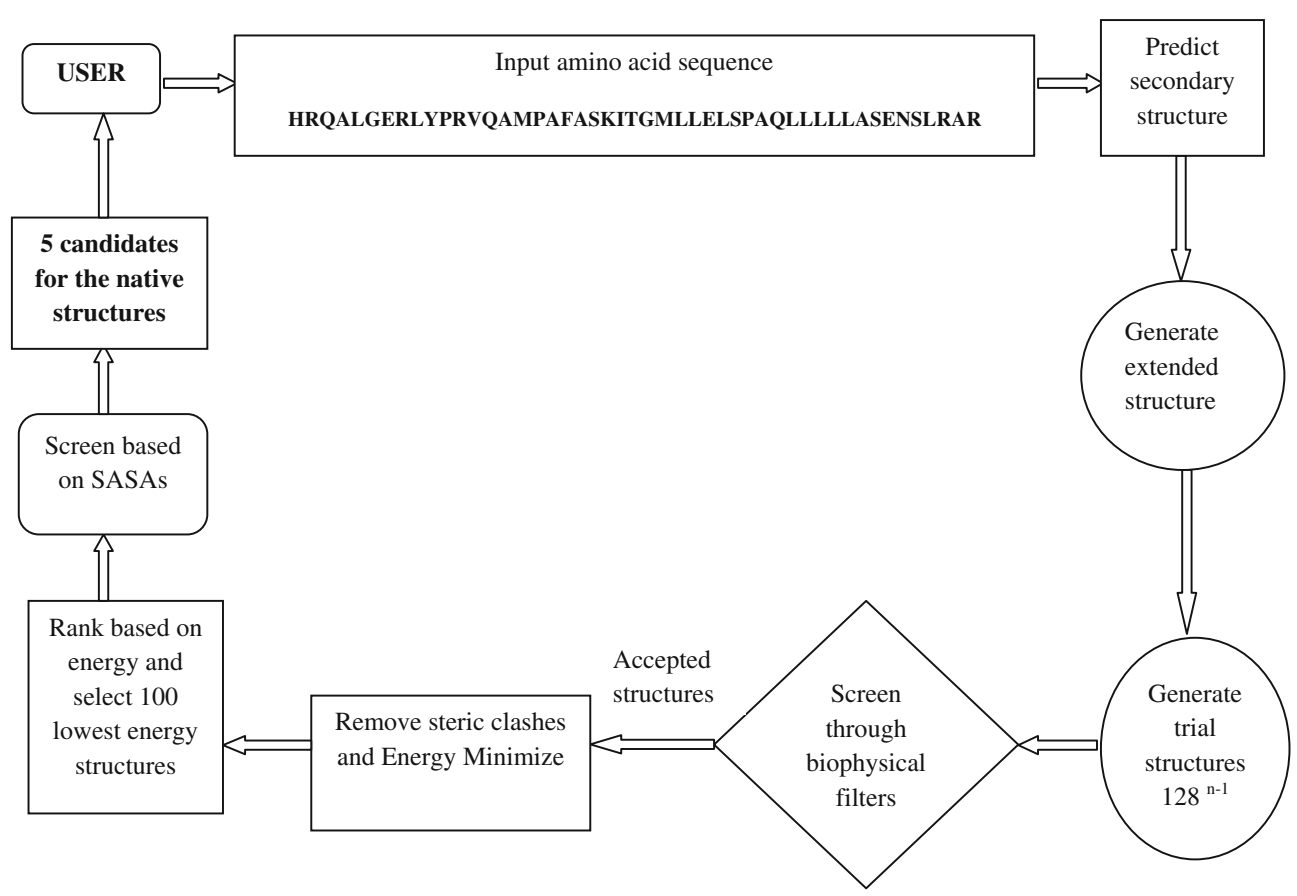

Figure 1. The flow of information in Bhageerath web server, starting with the input sequence from the user to the final prediction of five candidate structures for the native. 
gyration, topological distinctness of generated structures, inter-atomic distance and $\mathrm{C}_{\alpha}$ loop distance filters ${ }^{41}$ developed for the purpose of reducing the number of improbable candidates. The resultant structures are refined to the fifth module by a Monte Carlo sampling in dihedral space to remove steric clashes and overlaps involving atoms of main chain and side chains. In module six, the structures are energy minimized to further optimize the side chains. Module seven ranks the structures using an all atom energy based empirical scoring function ${ }^{42}$ and selects 100 lowest energy structures. Module eight reduces the structures selected in the previous module to five using solvent accessible surface areas (SASA). ${ }^{43}$ Short molecular dynamics simulations with explicit solvent for further refinement of the five structures is an optional last step. The protocol has been web-enabled and is freely accessible at http://www.scfbio-iitd.res.in/bhageerath. ${ }^{40}$ The flow chart diagram of Bhageerath is depicted in figure 1.

\section{Results and discussion}

The Bhageerath methodology has been validated on 80 small globular proteins ( $<100$ amino acids) consisting of up to five helices and strands with known tertiary structures. The results obtained for the 80 small globular proteins with the web server are shown in table 2 . For each of these proteins, a structure within $3-7 \AA$ RMSD (root mean square deviation) of the native has been obtained in the five lowest energy structures. Figure 2 shows a superimposition of the lowest RMSD predicted structure with the native structure for the 80 test proteins.

All the eight modules of the protocol are currently incorporated on a dedicated 280 AMD Opteron $2.4 \mathrm{GHz}$ processor cluster. In contrast to typical short return times (ranging from 1 to $10 \mathrm{~min}$ ) for receiving results from comparative (homology based) modelling servers, the expected prediction time with Bhageerath web server for small systems ( $\leq 3$ helices) is $\sim 10 \mathrm{~min}$. The prediction times in case of Bhageerath depend on the length of the sequence, number of secondary structural elements, number of trial structures generated and the accepted number of trial structures after biophysical filters which undergo all atom energy processing.

The earlier version of Bhageerath had a limitation of predicting structures of proteins with not more than 100 amino acids and 2-3 secondary structures. Pushing the frontiers of the atomic models, we have now developed a new methodology which can handle proteins with more than 100 amino acids. The protocol uses a 'divide and conquer' strategy based on the number of secondary structure elements, wherein the query sequence is divided into overlapping fragments and five structures are generated for each fragment with Bhageerath methodology as described above, followed by a patching of all the fragments and scoring them with a final selection of five structures for the overall sequence. This methodology is under rigorous validation.

\section{Development of Bhageerath-H}

The major obstacle in the computational structure prediction based on first principles is the conformational sampling. Sampling the entire conformational space of the polypeptide starting from a fully extended conformation and selecting a conformation which uniquely has a lower energy than the non-native conformations is a daunting task. The Bhageerath can predict structures of small proteins with an RMSD $<7-10 \AA$ from the native almost routinely now in an automated mode, but for larger proteins we envisage development of an $a b$ initio-homology hybrid methodology christened Bhageerath-H for tertiary structure prediction for improved accuracy. In Bhageerath-H methodology, we identify regions of polypeptide chain where local sequence similarities are realized, create 3D-structural fragments using conventional bioinformatics tools, use the $a b$ initio method for regions with no matches in structural databases, and patch

Table 2. Validation of Bhageerath Protocol on 80 small globular proteins.

\begin{tabular}{|c|c|c|c|c|c|}
\hline Sl. No. & PDBID & No. of amino acids & $\begin{array}{l}\text { No. of secondary } \\
\text { structural elements }\end{array}$ & Lowest RMSD $(\AA)$ & $\begin{array}{c}\text { Energy rank of lowest } \\
\text { structure in top } 5 \text { structures }\end{array}$ \\
\hline 1 & $1 E 0 Q$ & 17 & $2 \mathrm{E}$ & 2.5 & 2 \\
\hline 2 & $1 \mathrm{~B} 03$ & 18 & $2 \mathrm{E}$ & 4.4 & 2 \\
\hline 3 & $1 \mathrm{WQC}$ & 26 & $2 \mathrm{H}$ & 2.5 & 3 \\
\hline 4 & 1RJU & 36 & $2 \mathrm{H}$ & 5.9 & 4 \\
\hline 5 & 1EDM & 39 & $2 \mathrm{E}$ & 3.5 & 2 \\
\hline 6 & 1AB1 & 46 & $2 \mathrm{H}$ & 4.2 & 5 \\
\hline 7 & $1 \mathrm{BX} 7$ & 51 & $2 \mathrm{E}$ & 3.2 & 4 \\
\hline
\end{tabular}


Table 2. (continued)

\begin{tabular}{|c|c|c|c|c|c|}
\hline Sl. No. & PDBID & No. of amino acids & $\begin{array}{l}\text { No. of secondary } \\
\text { structural elements }\end{array}$ & Lowest RMSD $(\AA)$ & $\begin{array}{c}\text { Energy rank of lowest } \\
\text { structure in top } 5 \text { structures }\end{array}$ \\
\hline 8 & 1FME & 28 & $1 \mathrm{H}, 2 \mathrm{E}$ & 3.7 & 5 \\
\hline 9 & $1 \mathrm{ACW}$ & 29 & $1 \mathrm{H}, 2 \mathrm{E}$ & 5.3 & 3 \\
\hline 10 & 1AIL & 70 & $3 \mathrm{H}$ & 4.4 & 3 \\
\hline 11 & 1B6Q & 56 & $2 \mathrm{H}$ & 3.8 & 5 \\
\hline 12 & $1 \mathrm{ROP}$ & 56 & $2 \mathrm{H}$ & 4.3 & 2 \\
\hline 13 & $1 \mathrm{NKD}$ & 59 & $2 \mathrm{H}$ & 3.9 & 1 \\
\hline 14 & $1 \mathrm{RPO}$ & 61 & $2 \mathrm{H}$ & 3.8 & 2 \\
\hline 15 & 1QR8 & 68 & $2 \mathrm{H}$ & 3.9 & 4 \\
\hline 16 & $1 \mathrm{YRF}$ & 35 & $3 \mathrm{H}$ & 4.8 & 4 \\
\hline 17 & 1YRI & 35 & $3 \mathrm{H}$ & 4.6 & 3 \\
\hline 18 & 2ERL & 40 & $3 \mathrm{H}$ & 4 & 3 \\
\hline 19 & 1RES & 43 & $3 \mathrm{H}$ & 4.2 & 2 \\
\hline 20 & $1 G V D$ & 52 & $3 \mathrm{H}$ & 5.1 & 4 \\
\hline 21 & $1 \mathrm{DFN}$ & 30 & $3 \mathrm{E}$ & 5 & 1 \\
\hline 22 & 1Q2K & 31 & $1 \mathrm{H}, 2 \mathrm{E}$ & 4.8 & 4 \\
\hline 23 & $1 \mathrm{SCY}$ & 31 & $1 \mathrm{H}, 2 \mathrm{E}$ & 3.1 & 5 \\
\hline 24 & $1 \mathrm{XRX}$ & 34 & $1 \mathrm{E}, 2 \mathrm{H}$ & 5.6 & 1 \\
\hline 25 & $1 \mathrm{ROO}$ & 35 & $3 \mathrm{H}$ & 2.8 & 5 \\
\hline 26 & $1 \mathrm{MBH}$ & 52 & $3 \mathrm{H}$ & 4 & 4 \\
\hline 27 & 1HDD & 57 & $3 \mathrm{H}$ & 5.5 & 4 \\
\hline 28 & $1 \mathrm{BDC}$ & 60 & $3 \mathrm{H}$ & 4.8 & 5 \\
\hline 29 & 1DF5 & 68 & $3 \mathrm{H}$ & 3.4 & 1 \\
\hline 30 & 1QR9 & 68 & $3 \mathrm{H}$ & 3.8 & 2 \\
\hline 31 & 1VII & 36 & $3 \mathrm{H}$ & 3.7 & 2 \\
\hline 32 & 1BGK & 37 & $3 \mathrm{H}$ & 4.1 & 3 \\
\hline 33 & 1BHI & 38 & $1 \mathrm{H}, 2 \mathrm{E}$ & 5.3 & 2 \\
\hline 34 & $10 V X$ & 38 & $1 \mathrm{H}, 2 \mathrm{E}$ & 4 & 1 \\
\hline 35 & 1I6C & 39 & $3 \mathrm{E}$ & 5.1 & 2 \\
\hline 36 & $2 \mathrm{G} 7 \mathrm{O}$ & 68 & $4 \mathrm{H}$ & 5.8 & 2 \\
\hline 37 & $2 \mathrm{OCH}$ & 66 & $4 \mathrm{H}$ & 6.6 & 3 \\
\hline 38 & 1WR7 & 41 & $3 \mathrm{E}, 1 \mathrm{H}$ & 5.2 & 2 \\
\hline 39 & $2 \mathrm{~B} 7 \mathrm{E}$ & 59 & $4 \mathrm{H}$ & 6.8 & 4 \\
\hline 40 & $1 \mathrm{FAF}$ & 79 & $4 \mathrm{H}$ & 6.4 & 4 \\
\hline 41 & $2 \mathrm{CPG}$ & 43 & $1 \mathrm{E}, 2 \mathrm{H}$ & 5.3 & 2 \\
\hline 42 & 1DV0 & 45 & $3 \mathrm{H}$ & 5.1 & 4 \\
\hline 43 & 1IRQ & 48 & $1 \mathrm{E}, 2 \mathrm{H}$ & 5.5 & 3 \\
\hline 44 & $1 \mathrm{GUU}$ & 50 & $3 \mathrm{H}$ & 4.6 & 4 \\
\hline 45 & 1GV5 & 52 & $3 \mathrm{H}$ & 4.1 & 2 \\
\hline 46 & 1PRB & 53 & $4 \mathrm{H}$ & 6.9 & 4 \\
\hline 47 & $1 \mathrm{DOQ}$ & 69 & $5 \mathrm{H}$ & 6.8 & 3 \\
\hline 48 & 1I2T & 61 & $4 \mathrm{H}$ & 5.4 & 4 \\
\hline 49 & 2CMP & 56 & $4 \mathrm{H}$ & 5.6 & 1 \\
\hline 50 & 1X4P & 66 & $4 \mathrm{H}$ & 5.2 & 3 \\
\hline 51 & $1 \mathrm{GAB}$ & 53 & $3 \mathrm{H}$ & 4.9 & 1 \\
\hline 52 & $1 \mathrm{MOF}$ & 53 & $3 \mathrm{H}$ & 2.9 & 5 \\
\hline 53 & $1 \mathrm{ENH}$ & 54 & $3 \mathrm{H}$ & 4.6 & 3 \\
\hline 54 & 1IDY & 54 & $3 \mathrm{H}$ & 3.6 & 5 \\
\hline 55 & 1PRV & 56 & $3 \mathrm{H}$ & 5 & 5 \\
\hline 56 & 1BW6 & 56 & $4 \mathrm{H}$ & 4.2 & 1 \\
\hline 57 & $2 \mathrm{~K} 2 \mathrm{~A}$ & 70 & $4 \mathrm{H}$ & 6.1 & 1 \\
\hline 58 & 1TGR & 52 & $4 \mathrm{H}$ & 6.8 & 2 \\
\hline 59 & 2 V75 & 90 & $5 \mathrm{H}$ & 7 & 3 \\
\hline 60 & 1HNR & 47 & $2 \mathrm{E}, 2 \mathrm{H}$ & 5.2 & 2 \\
\hline 61 & $1 \mathrm{I} 5 \mathrm{X}$ & 61 & $3 \mathrm{H}$ & 3.6 & 3 \\
\hline 62 & $1 \mathrm{II} Y$ & 61 & $3 \mathrm{H}$ & 3.4 & 5 \\
\hline 63 & 1KU3 & 61 & $3 \mathrm{H}$ & 5.5 & 4 \\
\hline
\end{tabular}


Table 2. (continued)

\begin{tabular}{|c|c|c|c|c|c|}
\hline Sl. No. & PDBID & No. of amino acids & $\begin{array}{l}\text { No. of secondary } \\
\text { structural elements }\end{array}$ & Lowest RMSD $(\AA)$ & $\begin{array}{l}\text { Energy rank of lowest } \\
\text { structure in top } 5 \text { structures }\end{array}$ \\
\hline 64 & 1YIB & 61 & $3 \mathrm{H}$ & 3.5 & 5 \\
\hline 65 & $1 \mathrm{AHO}$ & 64 & $1 \mathrm{H}, 2 \mathrm{E}$ & 4.5 & 4 \\
\hline 66 & $2 \mathrm{KJF}$ & 60 & $4 \mathrm{H}$ & 5 & 4 \\
\hline 67 & 1RIK & 29 & $2 \mathrm{E}, 2 \mathrm{H}$ & 4.4 & 4 \\
\hline 68 & 1JEI & 53 & $4 \mathrm{H}$ & 5.8 & 5 \\
\hline 69 & $2 \mathrm{HOA}$ & 68 & $4 \mathrm{H}$ & 6.3 & 4 \\
\hline 70 & 2DT6 & 62 & $4 \mathrm{H}$ & 5.9 & 3 \\
\hline 71 & 2L37 & 43 & $2 \mathrm{H}$ & 3 & 1 \\
\hline 72 & 2PMR & 76 & $3 \mathrm{H}$ & 6.8 & 2 \\
\hline 73 & $1 \mathrm{I} 2 \mathrm{~T}$ & 61 & $4 \mathrm{H}$ & 5.7 & 2 \\
\hline 74 & 2PM1 & 30 & $3 E$ & 4.6 & 4 \\
\hline 75 & $2 \mathrm{CJJ}$ & 63 & $3 \mathrm{H}$ & 5.2 & 1 \\
\hline 76 & 1WY3 & 35 & $3 \mathrm{H}$ & 5 & 4 \\
\hline 77 & 1P9I & 31 & $1 \mathrm{H}$ & 1.7 & 1 \\
\hline 78 & 3NMD & 53 & $1 \mathrm{H}$ & 1.9 & 1 \\
\hline 79 & $2 \mathrm{~J} 15$ & 15 & $2 \mathrm{E}$ & 2.6 & 5 \\
\hline 80 & 3E21 & 40 & $3 \mathrm{H}$ & 5.5 & 5 \\
\hline
\end{tabular}

(E=Strand; H=Helix; RMSD=root mean square deviation from the crystal structure i.e., native reported in RCSB)

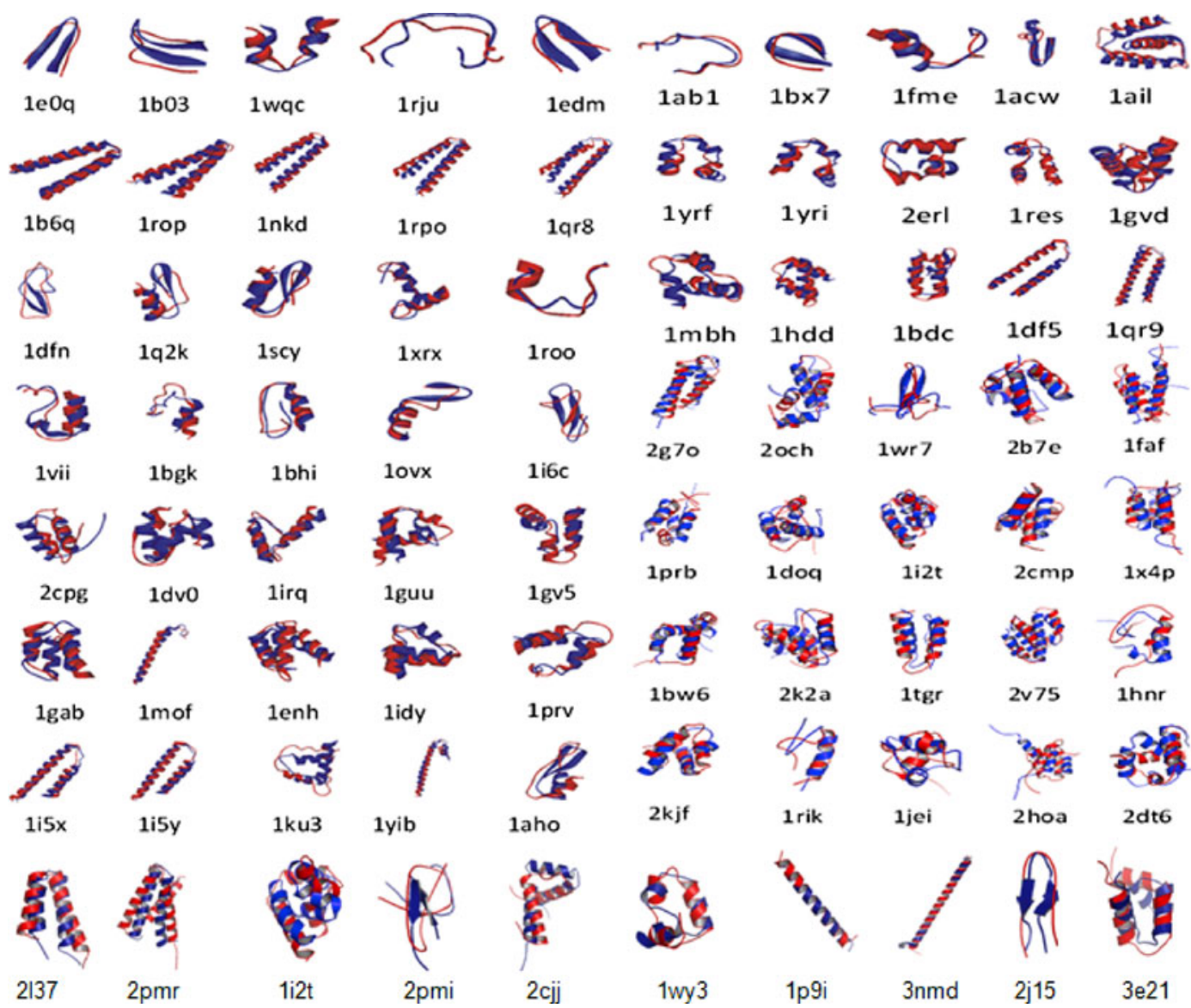

Figure 2. The superimposed lowest RMSD structure for the 80 small globular proteins. The PDB ID's are shown underneath each structure. The predicted structure is in red colour and the native (experimentally determined structure) is in blue. 


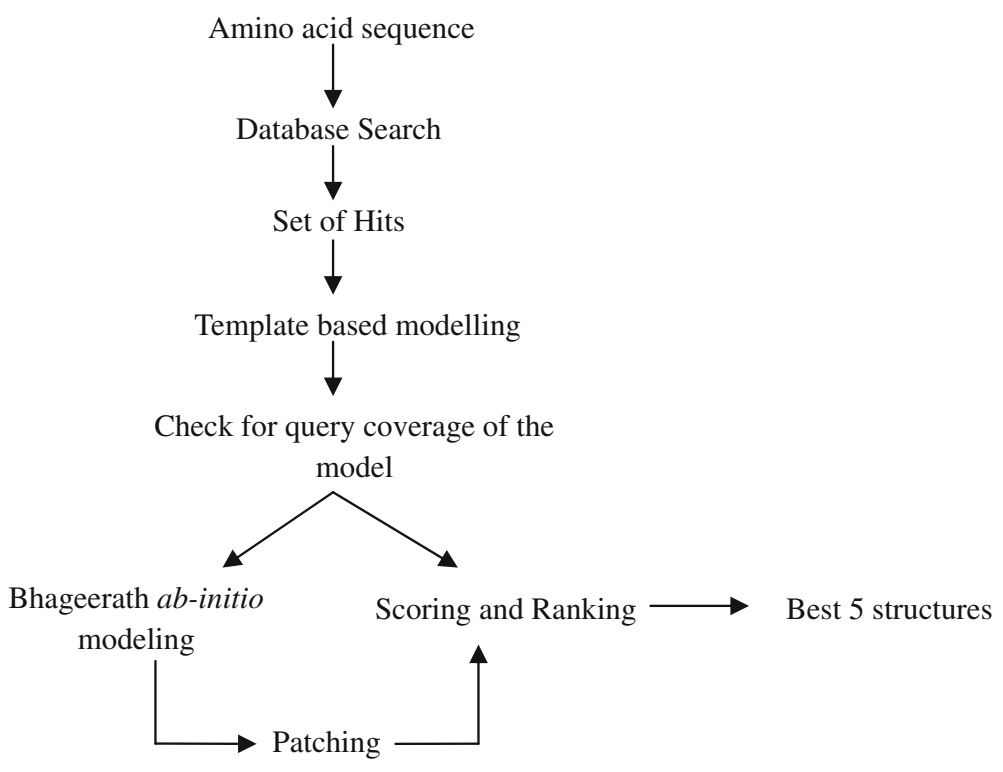

Figure 3. The flow chart of Bhageerath-H.

them to put together a complete structure for the proteins. Figure 3 shows a flow chart of Bhageerath-H methodology (http://www.scfbio-iitd.res.in/bhageerath/ bhageerath_h.jsp).

The protocol has been tested on various CASP9 targets of medium to large size (with more than 200 residues). Table 3 shows the root mean square deviation from the native of the best structures predicted by Bhageerath-H for five CASP9 targets.

A comparison of the structure predictions by Bhageerath, Bhageerath-H, Phyre2, ${ }^{44}$ ZhangServer, ${ }^{45,46}$ Baker-Rosetta ${ }^{47}$ and HHPredA ${ }^{48}$ for five CASP9 $^{49}$ targets was carried out (table 4). The table shows the RMSDs of the predictions by Bhageerath and Bhageerath-H in CASP9 and post-CASP9 and the RMSDs of the predictions submitted by four other servers in CASP9. In four of the five cases (T0538-D1, T0602-D1, T0605-D1, T0559-D1) Bhageerath was able to predict a structure within $10 \AA$ RMSD from the native with target T0643-D1 as an exception. Post

Table 3. Validation of the Bhageerath-H protocol on 5 CASP9 targets.

Sl. No. Target name No. of residues Lowest RMSD $(\AA)$

\begin{tabular}{llll}
\hline 1 & T0515 & 365 & 2.7 \\
2 & T0518 & 288 & 2.5 \\
3 & T0524 & 325 & 4.3 \\
4 & T0597 & 429 & 1.9 \\
5 & T0607 & 471 & 3.8
\end{tabular}

CASP9, we have incorporated a new version of structure generator and scoring function in Bhageerath- $H$, which has improved the prediction accuracy of the software (as seen in column 5). Excluding the templates with more than $30 \%$ similarity, the latest version of Bhageerath $-\mathrm{H}$ is able to predict a structure within $7 \AA$ RMSD from the native for all the 5 targets. In each of the five illustrative cases, Bhageerath and Bhageerath$\mathrm{H}$ are able to predict structures with RMSDs comparable to those obtained by some popular servers such as Phyre2, Zhang-server, Baker-Rosetta and HHPredA.

Thus, for sequences with known sequence homologs, Bhageerath-H has the potential to predict a structure with higher resolution, accuracy in less time. This clearly demonstrates the advantage of hybrid methods over $a b$ initio methods when a close homolog is available in the database, but for new sequences with no available sequence homologs, ab initio/de novo servers such as Bhageerath are the only alternative. Figure 4 shows a comparison of the structure prediction time and accuracies of Bhageerath and Bhageerath- $H$ software suites for small globular proteins.

\section{Conclusions}

We have described here an all atom energy based computational methodology, Bhageerath for tertiary structure prediction of small soluble proteins. Results on 80 globular proteins show that Bhageerath web server predicts one or more candidate structures within an RMSD of $7 \AA$ from the native for proteins with less 
Table 4. A comparison of protein tertiary structure prediction accuracies of Bhageerath and Bhageerath-H with Phyre2, Baker-Rosetta, Zhang-Server and HHpredA software for 5 CASP9 (3 May to 17 July, 2010) targets.

\begin{tabular}{|c|c|c|c|c|c|c|c|c|c|}
\hline Sl. No. & PDBID & Residues & CASP9 ID & $\begin{array}{c}\text { Lowest } \\
\text { RMSD (A) } \\
\text { prediction by } \\
\text { Bhageerath } \\
\text { post CASP9 } \\
\text { (in CASP9) }\end{array}$ & $\begin{array}{c}\text { Lowest } \\
\text { RMSD }(\AA) \\
\text { prediction by } \\
\text { Bhageerath-H } \\
\text { post CASP9 } \\
\text { (in CASP9) }\end{array}$ & $\begin{array}{c}\text { Lowest } \\
\text { RMSD }(\AA) \\
\text { prediction } \\
\text { by Phyre2 } \\
\text { in CASP9 }\end{array}$ & $\begin{array}{c}\text { Lowest } \\
\text { RMSD (A) } \\
\text { prediction by } \\
\text { Baker-Rosetta } \\
\text { Server } \\
\text { in CASP9 }\end{array}$ & $\begin{array}{c}\text { Lowest } \\
\text { RMSD }(\AA) \\
\text { prediction } \\
\text { by Zhang- } \\
\text { Server } \\
\text { in CASP9 }\end{array}$ & $\begin{array}{c}\text { Lowest } \\
\text { RMSD }(\AA) \\
\text { prediction } \\
\text { by } \\
\text { HHpredA } \\
\text { in CASP9 }\end{array}$ \\
\hline 1 & 2L09 & 53 & T0538-D1 & $6.88(9.02)$ & $1.87^{\#}$ & 2.11 & 2.17 & 1.39 & 2.15 \\
\hline 2 & $3 \mathrm{NKZ}$ & 55 & T0602-D1 & $3.43(4.71)$ & $2.90(2.90)$ & 2.14 & 1.61 & 1.49 & 2.14 \\
\hline 3 & 3NMD & 49 & T0605-D1 & $1.97(2.48)$ & $3.80(16.49)$ & 2.84 & 1.84 & 1.97 & 1.62 \\
\hline 4 & 3NZL & 73 & T0643-D1 & $4.81(10.31)$ & $3.49(4.59)$ & 5.19 & 5.68 & 4.30 & 8.9 \\
\hline 5 & 2L01 & 67 & T0559-D1 & $8.33(8.15)$ & $5.59^{\#}$ & 2.88 & 0.98 & 1.66 & 2.19 \\
\hline
\end{tabular}

\#These targets were fielded only for server prediction category and not for human expert group in CASP9.

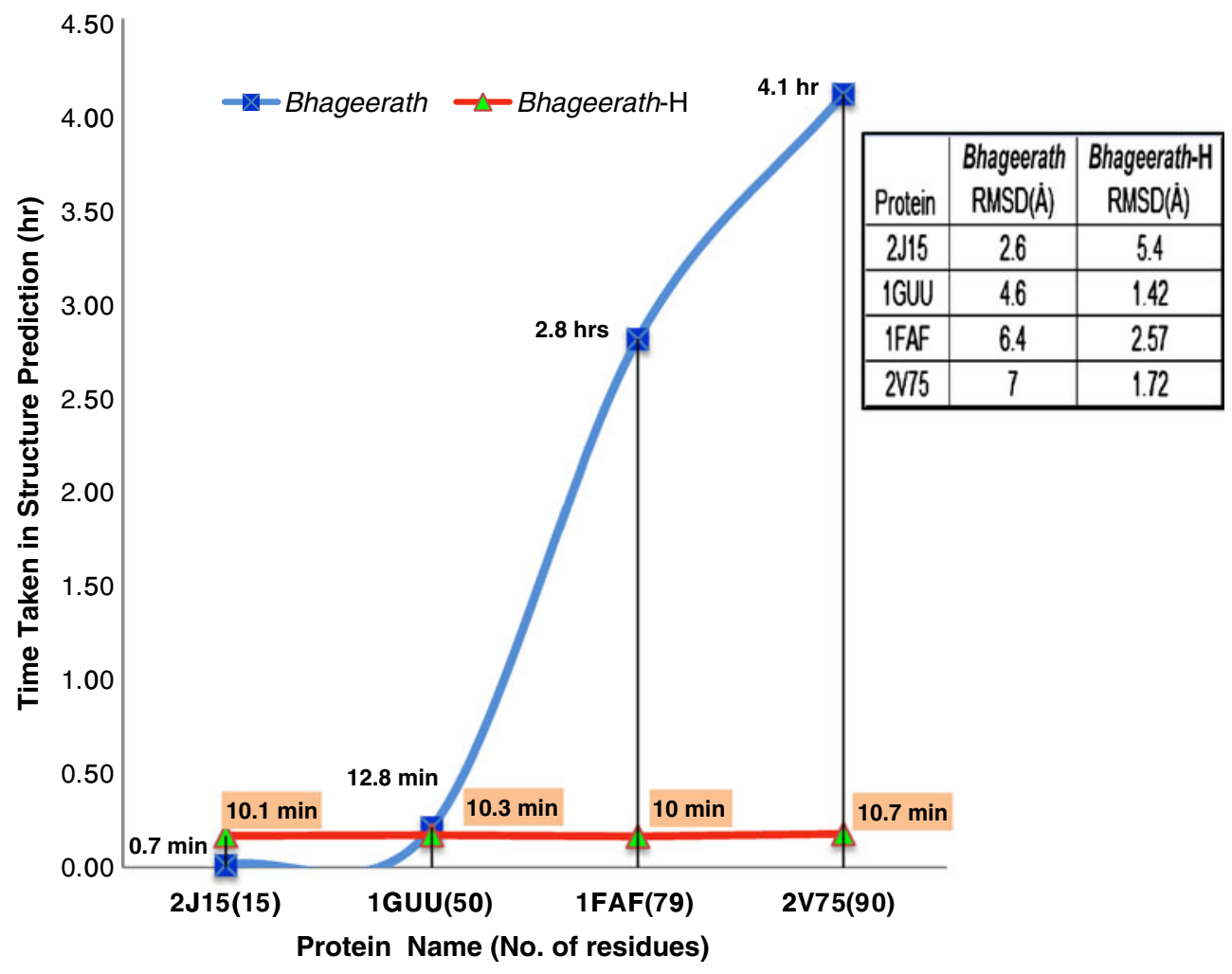

Figure 4. A comparison of the structure prediction time and accuracy of Bhageerath and Bhageerath-H software suites for four small globular proteins with $<100$ amino acids.

than five secondary structural elements. CASP9 results reflect the potential of the protocol to predict a structure within $10 \AA$ RMSD from the native for sequences with no known sequence homologs. For proteins with local sequence and structure matches involving short fragments, it is expedient to use a hybrid method such as Bhageerath- $\mathrm{H}$ for tertiary structure prediction. In a nutshell, for small proteins the structure prediction problem is under control with ab initio methods, and for larger proteins computational protocols involving hybrid models are getting better and better.

\section{Acknowledgements}

Funding from the Department of Information Technology and programme support from the Department 
of Biotechnology, Government of India to Supercomputing Facility for Bioinformatics are gratefully acknowledged.

\section{References}

1. Creighton T E 1990 Biochem. J. 2701

2. Dobson C M 2003 Nature 426884

3. Editorial 2005 Science $\mathbf{3 0 9} 78$

4. Unger R and Moult J 1993 Bull. Math. Biol. 551183

5. Fraenkel A S 1993 Bull. Math. Biol. 551199

6. Baker D 2000 Nature $\mathbf{4 0 5} 39$

7. Klepeis J L and Floudas C A 2004 SIAM News 371

8. Venkatraman J, Shankaramma S C and Balaram P 2001 Chem. Rev. 1013131

9. Lindorff-Larsen K, Piana S, Palmo K, Maragakis P, Klepeis J L, Dror R O and Shaw D E 2010 Proteins 78 1950

10. Levitt M and Warshel A 1975 Nature 253694

11. McCammon J A, Gelin B R and Karplus M 1977 Nature 267585

12. Li A and Daggett V 1995 Protein Eng. 81117

13. Daggett V and Levitt M 1992 Proc. Natl. Acad. Sci. USA 895142

14. Levitt M 1983 J. Mol. Biol. 168595

15. Levitt M 1983 J. Mol. Biol. 168621

16. Tirado-Rives J and Jorgensen W L 1993 Biochemistry 324175

17. Boczko E M and Brooks C L III 1995 Science 269393

18. Demchuk E, Bashford D and Case D A 1997 Fold. Des. 235

19. Daura X, Jaun B, Seebach D, van Gunsteren W F and Mark A E 1998 J. Mol. Biol. 280925

20. Duan Y and Kollman P A 1998 Science 282740

21. Mayor U, Johnson C M, Daggett V and Fersht A R 2000 Proc. Natl. Acad. Sci. USA 9713518

22. Zagrovic B, Sorin E J and Pande V S 2001 J. Mol. Biol. 313151

23. Simmerling C, Strockbine B and Roitberg A E 2002 J. Am. Chem. Soc. 12411258

24. Snow C D, Zagrovic B and Pande V S 2002 J. Am. Chem. Soc. 12414548

25. Freddolino P L, Liu F, Gruebele M and Schulten K 2008 Biophys. J. 94 L75

26. Freddolino P L, Park S, Roux B and Schulten K 2009 Biophys. J. 963772
27. Voelz V A, Bowman G R, Beauchamp K and Pande V S 2010 J. Am. Chem. Soc. 1321526

28. Shaw D E, Maragakis P, Lindorff-Larsen K, Piana S, Dror R O, Eastwood M P, Bank J A, Jumper J M, Salmon J K, Shan Y and Wriggers W 2010 Science 330 341

29. Allen F et al. 2001 IBM Syst. J. 40310

30. Shirts M and Pande V S 2000 Science 2901903

31. Liwo A, Khalili M and Scheraga H A 2005 Proc. Natl. Acad. Sci. USA 1022362

32. Ensign D L, Kasson P M and Pande V S 2007 J. Mol. Biol. 374806

33. Cooper S, Khatib F, Treuille A, Barbero J, Lee J, Beenen M, Leaver-Fay A, Baker D, Popovic Z and Players F 2010 Nature 466756

34. Bonneau R and Baker D 2001 Annu. Rev. Biophys. Biomol. Struct. 30173

35. Petrey D and Honig B 2005 Mol. Cell 20811

36. Moult J, Pedersen J T, Judson R and Fidelis K 1995 Proteins 23 ii

37. Moult J, Fidelis K, Kryshtafovych A, Rost B and Tramontano A 2009 Proteins 771

38. Berman H M, Westbrook J, Feng Z, Gilliland G, Bhat T N, Weissig H, Shindyalov I N, Bourne P E 2000 Nucleic Acids Res. 28235

39. Floudas C A, Fung H K, McAllister S R, Monnigmann M and Rajgaria R 2006 Chem. Eng. Sci. 61966

40. Jayaram B, Bhushan K, Shenoy S R, Narang P, Bose S, Agrawal P, Sahu D and Pandey V 2006 Nucleic Acids Res. 346195

41. Narang P, Bhushan K, Bose S and Jayaram B 2005 Phys. Chem. Chem. Phys. 72364

42. Narang P, Bhushan K, Bose S and Jayaram B $2006 J$. Biomol. Struct. Dyn. 23385

43. Hubbard S J and Thornton J M 1993 NACCESS Computer Program (London: Department of Biochemistry and Molecular Biology, University College London)

44. Kelley L A and Sternberg M J E 2009 Nature Protocols 4363

45. Roy A, Kucukural A and Zhang Y 2010 Nature Protocols 5725

46. Zhang Y 2008 BMC Bioinformatics 91

47. Kim D E, Chivian D and Baker D 2004 Nucleic Acids Res. 32 W526

48. Soding J, Biegert A and Lupas A N 2005 Nucleic Acids Res. 33 W244

49. http://predictioncenter.org/casp9/ 